\title{
Comparison of models to identify thermal characteristics of multi-layer building walls using inverse methods
}

\author{
Manon Rendu ${ }^{1,2}$, Jérôme Le Dréau ${ }^{1,2}$, Patrick Salagnac ${ }^{1,2}$, Maxime Doya ${ }^{3}$, \\ Mathilde Colmet Daâge ${ }^{2,4}$ \\ ${ }^{1}$ LaSIE, La Rochelle University, CNRS, UMR 7356, La Rochelle, France \\ ${ }^{2} 4$ evLab, La Rochelle University, CNRS, Electricité de France EDF, La Rochelle, France \\ ${ }^{3}$ TIPEE Platform, Lagord, France \\ ${ }^{4}$ EDF R\&D - ENERBAT, EDF Lab Les Renardières, Moret-sur-Loing, France
}

\begin{abstract}
The objective of this study was to evaluate inverse techniques for estimating the resistance and the capacity of walls in real weather conditions. The inverse techniques were applied on temperatures measured by sensors located in the wall. The performance of four different models were compared.

We demonstrated a high correlation with some parameters. A solution was proposed to limit the number of parameters identified, while allowing the identification of the thermal resistance and thermal capacity of the walls. The high uncertainty applied to some experimental data led to the exclusion of these data from the inverse method to improve its convergence.

Most of the models were highly accurate in estimating thermal resistance and capacity of a lightweight wall using a set of experimental data.
\end{abstract}

\section{Nomenclature}

a Thermal diffusivity, $\mathrm{m}^{2} \cdot \mathrm{s}^{-1}$

c Specific heat capacity, $\mathrm{J}_{\mathrm{kg}} \mathrm{kg}^{-1} \mathrm{~K}^{-1}$

C Total thermal mass, $\mathrm{J} \cdot \mathrm{m}^{-2} \cdot \mathrm{K}^{-1}$

e Thickness, $\mathrm{m}$

$\mathrm{P}$ Response factor of solar radiation excitation

$\mathrm{p} \quad$ Laplace variable

Q Heatflux, W.m-2

$\mathrm{R}$ Thermal resistance, $\mathrm{m}^{2} . \mathrm{K} . \mathrm{W}^{-1}$

$\mathrm{t}$ Time, $\mathrm{s}$

T Temperature, ${ }^{\circ} \mathrm{C}$

$\mathrm{X}$ Response factor of indoor temperature excitation

$\mathrm{Z} \quad$ Response factor of outdoor temperature excitation

Greek symbols:

$\alpha$ Absorptivity

$\lambda$ Thermal conductivity, W. $\mathrm{m}^{-1} \cdot \mathrm{K}^{-1}$

$\theta \quad$ Temperature in Laplace domain, ${ }^{\circ} \mathrm{C}$

$\varphi \quad$ Heatflux in Laplace domain, W. $\mathrm{m}^{-2}$

$\rho \quad$ Density, $\mathrm{kg} \cdot \mathrm{m}^{-3}$

Index and exponent:

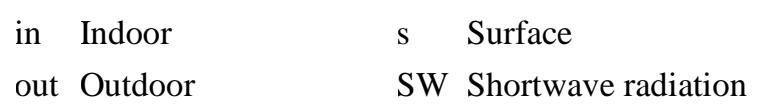

\section{Introduction}

Of all the factors that influence the energy consumption of buildings, one of the most important is the overall energy performance of the building envelope, mainly because of the heat loss by conduction through opaque walls and windows, air leakages (Meiss and Feijo-Munoz, 2015), and also, because of the degradation of its performance through time (Eleftheriadis and Hamdy, 2017).

The heat loss by conduction can be characterized by the physical properties of each material constituting the wall, such as $\lambda, c$ and $\rho$. From these physical and geometrical properties, it is possible to determine the $R$-value and $C$ value of the wall components (Asan and Sancaktar 1998). The material properties are usually evaluated in a laboratory using standard tests as described in (NF EN $12939,2000)$ and (EN 12667, 2001). The main drawback of this method is that it cannot be applied on whole implemented building walls.

In order to measure the thermal characteristics of the whole envelope already built or under test, new methods have been developed such as the heat flux meter method (ISO 9869-1, 2014), which consists in calculating the thermal transmittance of walls using three sensors: two temperature sensors (inside and outside) and a heat flux meter. The main drawbacks are that the method takes days or even weeks and the thermal capacity cannot be estimated. Quicker methods such as the dynamic method (ISO 9869-1, 2014) and ARX-modelling are described in (Deconinck and Roels, 2016) and (Gaspar et al., 2016).

The aim of this paper is to compare different models, such as Resistance-Capacitance models, response factor models and quadrupole models in order to determine which one is the most appropriate to identify the thermal characteristics of walls for different types of envelope (lightweight and heavyweight walls). A way to obtain this information is to apply inverse techniques (Beck and Arnold, 1977; Beck et al., 1985) considering a model, boundary conditions and temperature sensors data using a minimization algorithm. Identifying $\lambda$ and $\rho . c$ of the materials of the wall allows the calculation of $R$-value and $C$-value, which describe the thermal behaviour of an opaque wall.

In this article, the models (Resistance-Capacitance models, quadrupole models and response factor models) are described and the lightweight and heavyweight walls 
are briefly presented. Then, the models are validated numerically using real boundary conditions. Next, identification techniques are applied on simulated experimental data, thus allowing an estimation of uncertainties. Finally, identification techniques are applied on experimental data in order to assess the resistance and the thermal mass of the walls.

\section{Modelling}

Heat transfer through multi-layer walls are modelled on the heat equation:

$$
\frac{\partial T}{\partial t}-a \nabla^{2} T=0
$$

The boundary conditions are outdoor and indoor temperatures $\left(T_{\text {out }}\right.$ and $\left.T_{\text {in }}\right)$ and shortwave solar radiation $\left(Q_{S W}\right)$ normal to the surface. Theses boundary conditions are characterized by the internal and external convective and radiative heat transfer coefficients $\left(R_{\text {in }}=0.125\right.$ $\mathrm{m}^{2} . \mathrm{K}^{-\mathrm{W}^{-1}}$ and $\left.R_{\text {out }}=0.04 \mathrm{~m}^{2} . \mathrm{K} . \mathrm{W}^{-1}\right)$ and the exterior solar surface absorptivity for short wave radiations $(\alpha=0.24)$.

The case studies illustrated in Figure 1 are composed of three different materials, with constant properties. Temperature sensors are located outside of the boundary layer, at the surface and within the wall. They are used to identify the thermal properties.

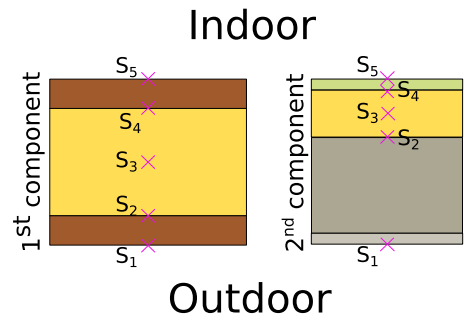

Figure 1: Sensor locations in the walls.

\section{Quadrupole model}

The thermal quadrupole model (the $Q R$ model) was developed in the 1980s. As mentioned in (Maillet et al., 2000) the "quadrupole method was developed as a tool for developing new techniques of thermal property measurements [...]. The quadrupole method allows the construction in a very simple way, of an exact and reliable direct model that can be used in the optimal design of the experiment as well as in the 'best fit' inversion process ». In this work, the second possible use of this method is considered.

In Figure 2, a quadrupole model of a three-layer model is presented.

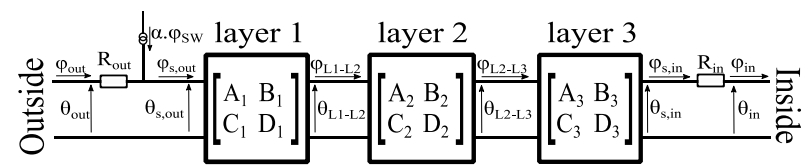

Figure 2: Quadrupole model structure.

Each layer $i$ is symbolized by a matrix.

$$
M_{i}=\left[\begin{array}{ll}
A_{i} & B_{i} \\
C_{i} & D_{i}
\end{array}\right]
$$

where $\left\{\begin{array}{c}A_{i}=D_{i}=\cosh \left(e_{i} \sqrt{p / a_{i}}\right) \\ B_{j}=\frac{\sinh \left(e_{i} \sqrt{p / a_{i}}\right)}{\lambda_{j} \sqrt{p / a_{i}}} \\ C_{j}=\lambda_{i} \sqrt{p / a_{i}} \sinh \left(e_{i} \sqrt{p / a_{i}}\right)\end{array}\right.$

The boundary conditions are taken into consideration as shown in (Maillet et al., 2000).

\section{Resistance-Capacitance model}

The Resistance-Capacitance model (the $R C$ model) considers walls as resistances and capacitances. Here, the nodes located at the interface between layers $i$ and $j\left(T_{L i-}\right.$ $\left.{ }_{L j}\right)$ or at the surface $\left(T_{s, \text { out }}\right.$ and $\left.T_{s, i n}\right)$ have no capacity. Capacities and resistances are equally distributed in each layer.

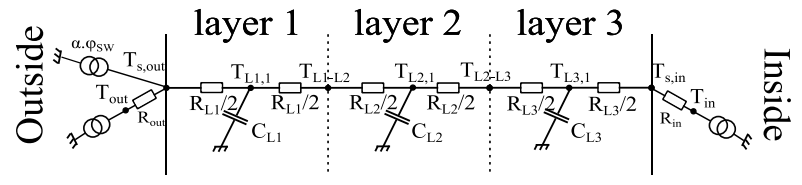

Figure 3: RC model structure for the model 6R3C.

If a simple RC model is considered (Figure 3), with one capacity in each layer, the following equations are obtained:

$\left(\begin{array}{c}0 \\ C_{L 1} \\ 0 \\ C_{L 2} \\ 0 \\ C_{L 3} \\ 0\end{array}\right) \cdot\left(\begin{array}{c}T_{s, \text { out }} \cdot \\ T_{L 1,1}^{\cdot} \\ T_{L 1-L 2} \\ T_{L 2,1}^{\cdot} \\ T_{L 2-L 3} \\ T_{L 3,1}^{\cdot} \\ T_{S, \text { in }}^{\cdot}\end{array}\right)=\left(\begin{array}{ccc}R_{\text {out }}{ }^{-1} & 0 & \alpha \\ 0 & \vdots & 0 \\ \vdots & & \vdots \\ & & \\ \vdots & 0 & \vdots \\ 0 & R_{\text {in }}{ }^{-1} & 0\end{array}\right) \cdot\left(\begin{array}{c}T_{\text {out }} \\ T_{\text {in }} \\ Q_{S W}\end{array}\right)+$

$$
\left.\begin{array}{ccccccc}
-\frac{1}{R_{\text {in }}}-\frac{2}{R_{1}} \frac{2}{R_{1}} & 0 & \cdots & \cdots & \cdots & 0 \\
\frac{2}{R_{1}} & \frac{-4}{R_{1}} & \frac{2}{R_{1}} & \ddots & & & \vdots \\
0 & \frac{2}{R_{1}}-\frac{2}{R_{1}}-\frac{2}{R_{2}} \frac{2}{R_{2}} & \ddots & & \vdots \\
\vdots & \ddots & \frac{2}{R_{2}} & \frac{-4}{R_{2}} & \frac{2}{R_{2}} & \ddots & \vdots \\
\vdots & & \ddots & \frac{2}{R_{2}}-\frac{2}{R_{1}}-\frac{2}{R_{2}} \frac{2}{R_{3}} & 0 \\
\vdots & & & \ddots & \frac{2}{R_{3}} & \frac{-4}{R_{3}} & \frac{2}{R_{3}} \\
0 & \cdots & \cdots & \cdots & 0 & \frac{2}{R_{3}}-\frac{1}{R_{\text {out }}}-\frac{2}{R_{3}}
\end{array}\right) \cdot\left(\begin{array}{c}
T_{s, \text { out }} \\
T_{L 1,1} \\
T_{L 1-L 2} \\
T_{L 2,1} \\
T_{L 2-L 3} \\
T_{L 3,1} \\
T_{s, \text { in }}
\end{array}\right)
$$

where $C$ is the matrix of capacities, $A$ is the matrix of resistances; $B$ and $U$ are matrices and vectors of boundary conditions; $T$ is the vector of temperatures.

In this paper, the method is applied on two RC models: the first one is called the 6R3C model (one capacity in each layer) and the second one is called the 18R15C model (five capacities in each layer).

\section{Response factor model}

Response factor models (the RF model) are described in (Mitalas and Stephenson, 1967).

The objective is to precisely calculate the response given by the simple excitation (triangular pulse) of each boundary condition using a well-described model (here, we used a RC model with a number of capacities higher than 10 per layer). In this case, the temperature response is called factor response for indoor temperature, outdoor temperature or solar radiation. An example is illustrated 
in Figure 4. Response factors are calculated for each boundary condition regarding sensor between the first and the second layer $\left(T_{L 1-L 2}\right)$ i.e, $X_{L 1-L 2}, Z_{L 1-L 2}$, and $P_{L 1-L 2}$.

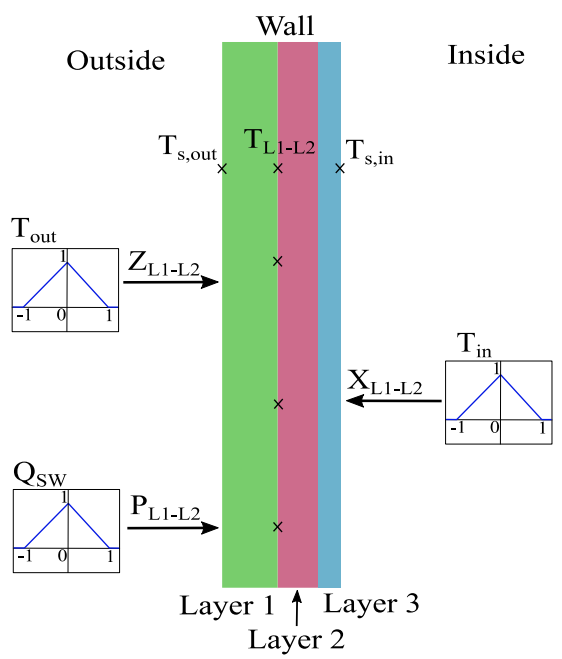

Figure 4: Response factor model description.

Once these response factors obtained and the real boundary conditions broken down into a series of triangular functions using a time step $\Delta$, it is possible to express each temperature as follows:

$T_{i}(t)=\sum_{j=0}^{n} Z_{i, j} * T_{\text {out }}(t-j \Delta)+\sum_{j=0}^{n} X_{i, j} * T_{i n}(t-$ $j \Delta)+\sum_{j=0}^{n} P_{i, j} * Q_{S W}(t-j \Delta)$

where $*$ is the convolution function.

This method is applied to sensors located within the wall and at the surface.

\section{Description of the walls and sensors}

The four models described before were applied on two case studies. The first one is related to a lightweight wall installed on a test cell located in TIPEE in La Rochelle, France. The second one is an insulated heavyweight wall located in BESTLab, EDF Lab, near Paris, France.

\section{The lightweight wall}

The lightweight wall under test is a wall made up of three homogeneous layers. Its layers and their material's properties are described in Table 1. Only one-dimensional heat transfer is considered.

Table 1: Description of the lightweight wall.

\begin{tabular}{|c|c|c|c|c|c|}
\hline \multicolumn{2}{|c|}{ Layers } & \multicolumn{5}{c|}{ Properties } \\
\hline Material & Code & $\lambda$ & $\rho$ & $c$ & $e$ \\
\hline Outside & & & & \\
\hline Plywood & $\mathrm{pl}$ & 0.13 & 650 & 1700 & 0.021 \\
\hline Polyurethane & $\mathrm{PUR}$ & 0.023 & 35 & 1000 & 0.12 \\
\hline Plywood & $\mathrm{pl}$ & 0.13 & 650 & 1700 & 0.021 \\
\hline Inside
\end{tabular}

From these data, it is possible to calculate the theoretical $R$-value and $C$-value of this wall:

$$
\begin{gathered}
R=\sum_{i} \frac{e_{i}}{\lambda_{i}} \\
C=\sum_{i} e_{i} \rho_{i} c_{i}
\end{gathered}
$$

For the lightweight wall, $R_{\text {Light }}=5.5 \mathrm{~m}^{2} . \mathrm{K} \mathrm{W}^{-1}$ and $C_{\text {Light }}=51 \mathrm{~kJ} \cdot \mathrm{m}^{-2} \cdot \mathrm{K}^{-1}$.

\section{The heavyweight wall}

The heavyweight wall under test is a wall made up of three homogeneous layers. Its layers and their materials' properties are described in Table 2 . Only one-dimensional heat conduction is considered.

Table 2: Description of the heavyweight wall.

\begin{tabular}{|l|c|c|c|c|c|}
\hline \multicolumn{1}{|c|}{ Layers } & \multicolumn{5}{|c|}{ Properties } \\
\hline \multicolumn{1}{|c|}{ Material } & Code & $\lambda$ & $\rho$ & $c$ & $e$ \\
\hline Outside \\
\hline Coating+Concrete & conc & 1.0 & 639 & 920 & 0.21 \\
\hline EPS & EPS & 0.04 & 14 & 1400 & 0.1 \\
\hline Gypsum & gyps & 0.31 & 832 & 800 & 0.01 \\
\hline Inside
\end{tabular}

Using equation 6: $R_{\text {Heavy }}=2.7 \mathrm{~m}^{2} \cdot \mathrm{K} \cdot \mathrm{W}^{-1}$ and $C_{\text {Heavy }}=$ $131 \mathrm{~kJ} \cdot \mathrm{m}^{-2} \cdot \mathrm{K}^{-1}$.

\section{Temperature sensors}

As shown in Figure 1, temperature sensors (4-wire resistance thermometers) were installed inside the components and on the internal and external surfaces.
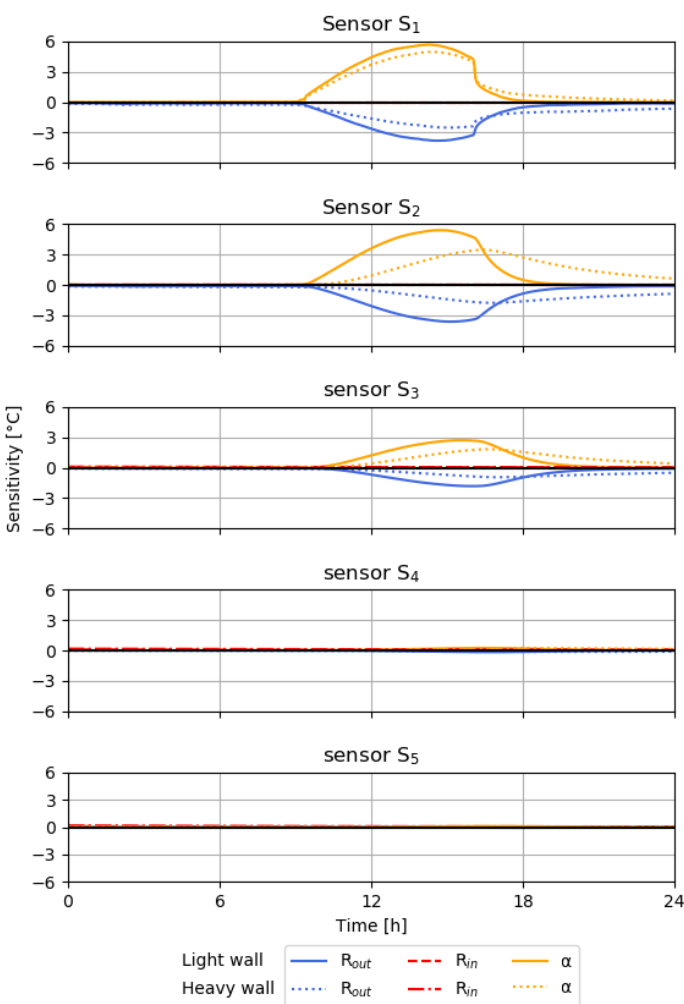

Figure 5: Sensitivity analysis of model 18R15C during a sunny winter's day-parameters $R_{\text {out }}, R_{\text {in }}$ and $\alpha$.

There were five sensors:

- Sensor $S_{1}$, on the external surface,

- Sensor $S_{2}$, between the external layer and the insulation,

- Sensor $\mathrm{S}_{3}$, in the middle of the insulation,

- Sensor $\mathrm{S}_{4}$, between the internal layer and the insulation,

- Sensor $\mathrm{S}_{5}$, on the internal surface.

Among these sensor locations, some of them were highly dependent on the modelling hypothesis since the 
uncertainty on several parameters was high, such as $R_{i n}$, $R_{\text {out }}$ and $\alpha$.

$R_{\text {in }}$ and $R_{\text {out }}$ are standard values defined in (NF EN ISO 6946, 2017). According to Annex A of this standard, $R_{\text {in }}$ varies between 0.11 and $0.13 \mathrm{~m}^{2} . \mathrm{K} / \mathrm{W}$ between 20 and $50^{\circ} \mathrm{C}$ and $R_{\text {out }}$ varies between 0.04 and $0.06 \mathrm{~m}^{2} . \mathrm{K} / \mathrm{W}$ when the wind speed varies between 2 and $6 \mathrm{~m} / \mathrm{s} . \alpha$ is a property derived from a measure performed on a spectrophotometer with an integrating sphere. $\alpha$ varies between 0.18 and 0.35 depending on the cleanliness of the material and the solar spectral distribution.

Figure 5 shows a sensitivity analysis that takes into account these possible variations during a sunny winter's day. It seems clear that sensor $S_{1}$ and $S_{2}$ are highly sensitive to $\alpha$ and $R_{\text {out }}$ variations for both walls. However, in the case of the heavyweight wall, the impact is reduced. It was preferable to reject the results from sensors $S_{1}$ and $\mathrm{S}_{2}$ as $R_{\text {out }}$ and $\alpha$ were not known with great accuracy. The uncertainty could have had a negative impact on the identification. Sensor $S_{5}$ were also excluded since measurement of the surface temperatures is more challenging and bear a higher experimental uncertainty. In fact, the temperature difference between the air and the wall surface is so small that it is lower than the temperature measurement uncertainty $\left( \pm 0.15^{\circ} \mathrm{C}\right)$ most of the time. Only sensors $S_{3}$ and $S_{4}$ are selected for identification.

\section{Model validation}

The models were validated for cases encountered in experimental tests. These cases were classified into two categories for the winter season: cloudy days and sunny days. A model was validated if it succeeded in accurately reproducing the thermal behaviour of the wall during these two typical days.

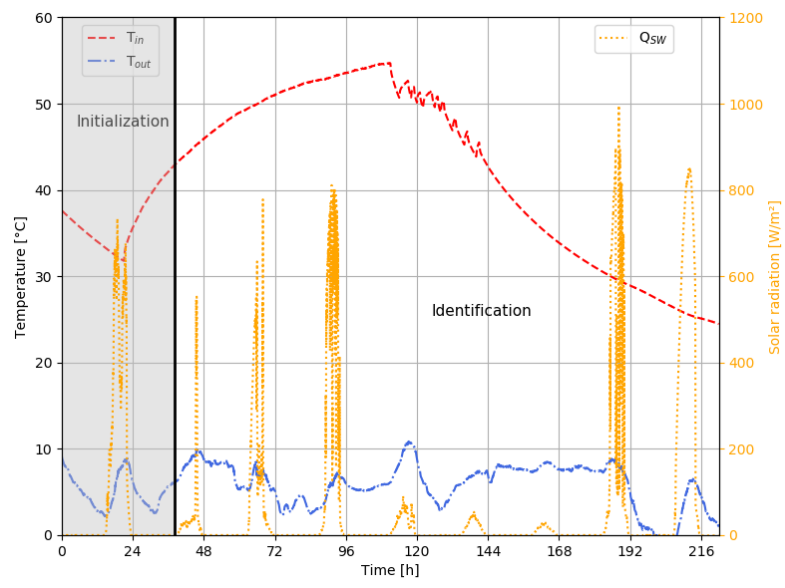

Figure 6: Boundary conditions for ROLBS case.

\section{Boundary conditions}

The typical sunny and cloudy winter's days came from a larger case, called the ROLBS case, as shown in the Figure 6 . Real boundary conditions were measured by a weather station located near La Rochelle, France. The ROLBS case lasted 9 days during the winter season with a measurement time step of 120 s.

This case was used to identify the $R$ - and $C$-values numerically and experimentally as described below.

As illustrated in Figure 7, during the cloudy winter's day, indoor temperature was almost constant. Outdoor temperature and solar radiation were those of a cloudy winter's day. During the sunny winter's day, indoor temperature was almost constant. Outdoor temperature and solar radiation were those of a sunny winter's day. Both cases lasted $24 \mathrm{~h}$ with a measurement time step of 120 s.
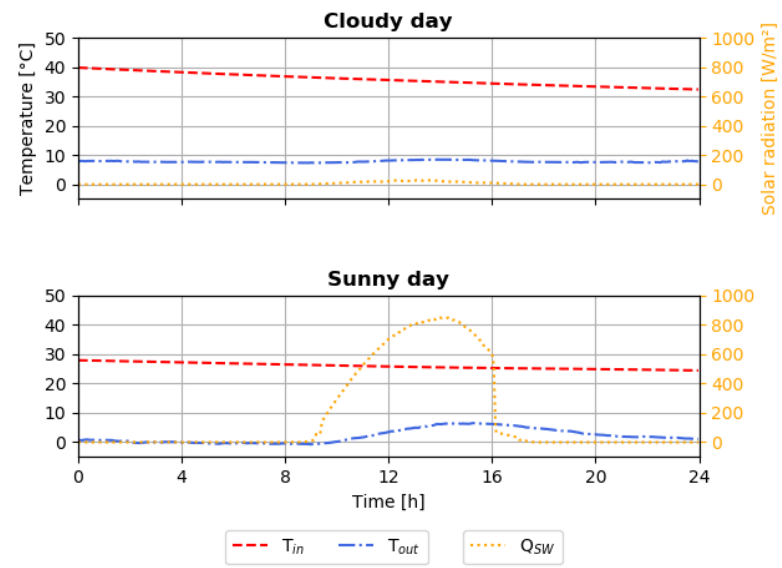

Figure 7: Boundary conditions for the cloudy and the sunny winter's day.

The initial conditions were the temperature values obtained assuming that the steady state was reached at the first time step.

\section{Validation of the direct models for the lightweight wall}

The results obtained by simulation of the $6 R 3 \mathrm{C}$ model, the $18 R 15 C$ model, the QR model and the RF model, i.e. the reduced models were compared to the results obtained with finite element calculation using Comsol@ software (COMSOL, 2019), namely the simulated experimental data.

The comparison of models was based on the study of their residuals, i.e. the absolute value of the difference between the simulated experimental data and each reduced model. The means of residuals of each model for a cloudy day and for a sunny day for the lightweight wall are presented in Figure 8.

Regarding the results for the $R C$ models, it seems obvious that the more capacities there are, the lower the residuals are.

Almost all the residuals are under $0.1^{\circ} \mathrm{C}$ for both sensors. The residuals for sensor $\mathrm{S}_{3}$ are higher for the 6R3C model.

Best models are the 18R15C model, the RF model and the $Q R$ model since their residuals are the lowest, mostly for sensor $\mathrm{S}_{3}$. 

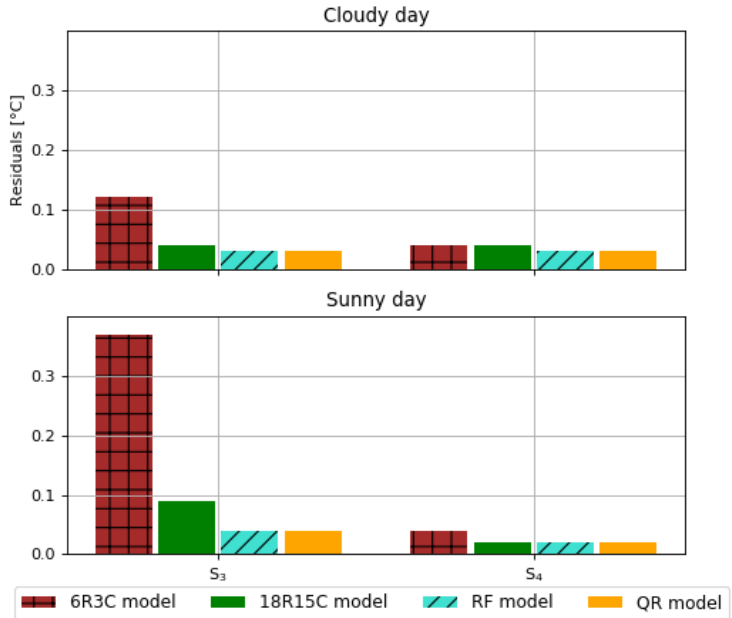

Figure 8: Mean of residuals of each model for sensors $S_{3}$ and $S_{4}$.

Validation of the direct models for the heavyweight wall

The same method was applied to the heavyweight wall. The results are presented in Figure 9.
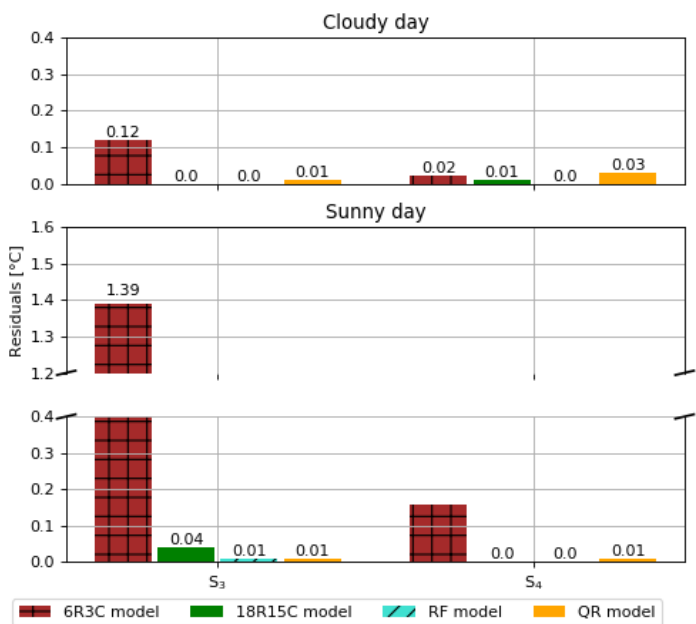

Figure 9: Mean of residuals of each model for sensors $S_{3}$ and $S_{4}$.

Residuals of the 6R3C model are extremely high. This can be explained by the fact that this model is not able to simulate high capacities and that sensor $\mathrm{S}_{3}$ is the sensor that is the most affected by the solar radiations since it is located closer to the external surface. Regarding the three other models, residuals are close to the residuals calculated for the lightweight wall.

As a conclusion of this validation part, the forecasts of the $6 R 3 C$ model are far from expected values. Consequently, the $6 R 3 C$ model is not validated.

\section{Identification}

\section{Identification technique}

On the one hand, measured values were available (simulated experimental data or experimental data) and on the other hand, outputs from the reduced models defined above were generated. The aim was to find the values of the physical parameters entered in the models so that the outputs from the reduced models were as close as possible to the experimental values.

The inverse method consists in minimizing the objective function defined as:

$$
f_{o b j}=\frac{1}{n * m} \sum_{j=1}^{m} \sum_{i=1}^{n}\left(T_{i, j}^{\text {sensor }}-T_{i, j}^{\text {model }}\right)^{2}
$$

Where $m$ is the number of time steps, $n$ the number of sensor, $T_{i, j}^{\text {sensor }}$ is simulated experimental data or experimental data and $T_{i, j}^{\text {model }}$ is data obtained from the chosen model among the 18R15C modes, the RF model and the QR model.

The Nelder-Mead algorithm was used to minimize this function (Nelder and Mead, 1965). The function optimize.minimize in Scipy Python's library was used.

The physical parameters ( $\lambda$ and $\rho . c$ ) of each layer were estimated. Then, it was possible to calculate the $R$ - and $C$ values of the studied wall with equation 6 .

In order to avoid local minima, different initial values were used.

\section{Sensitivity analysis}

As shown by (Remy and Degiovanni, 2005), minimizing $f_{o b j}$ is equivalent to calculate its derivatives and find when it is equal to zero. The derivatives are called the sensitivity coefficients. A sensitivity analysis was carried out to study the variation of the response of sensors regarding the variation of unknown physical parameters ( $\lambda$ and $\rho . c$ ).
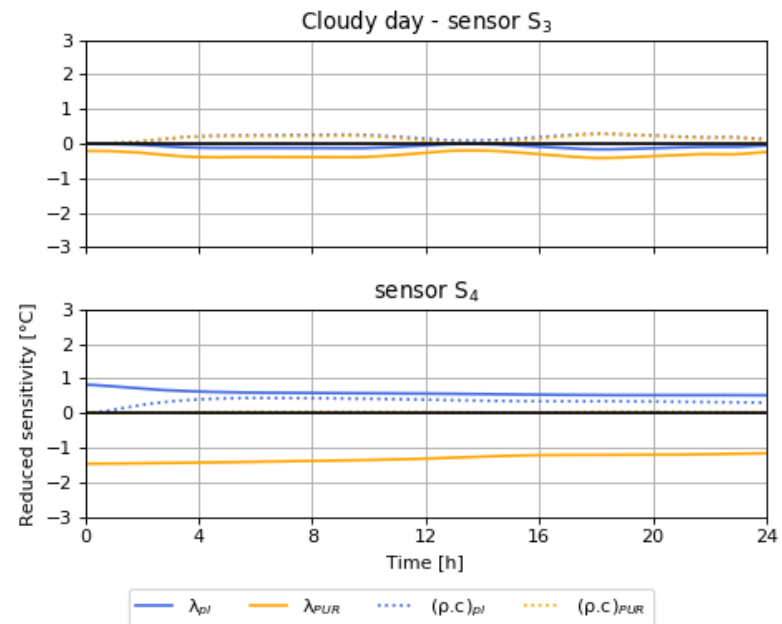

Figure 10: Sensitivity analysis of the 18R15C model for the lightweight wall during the cloudy day.

In the case of the lightweight wall, variations of $\lambda_{p l}, \lambda_{P U R}$, $(\rho . c)_{p l}$ and $(\rho . c)_{P U R}$ were studied. The reduced sensitivities against time are plotted in Figure 10 and Figure 11 for the 18R15C model during the cloudy and the sunny day described above. The results obtained for other models are similar since all models are based on Equation 1.

It seems clear that the reduced sensitivity is high for the four thermal properties, mainly for sensor $\mathrm{S}_{3}$. The dataset for identification should therefore contain sunny days to identify both $R$ - and $C$-values. 

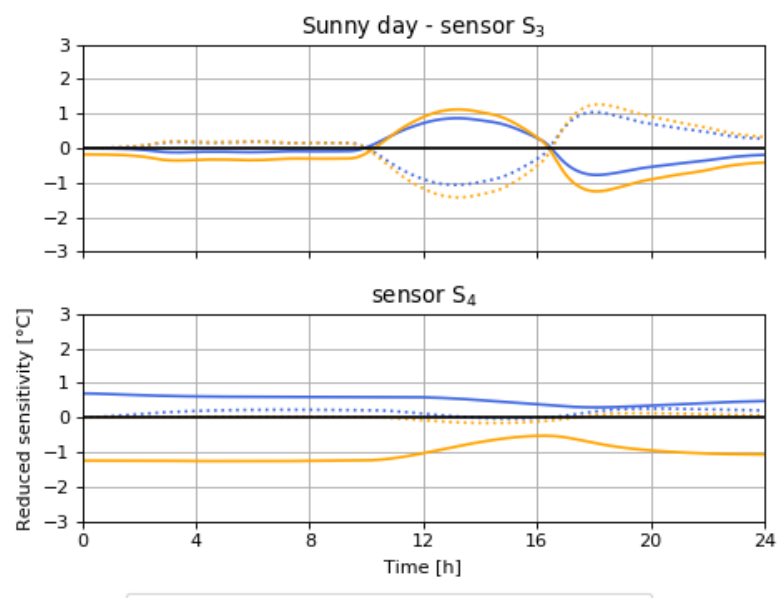

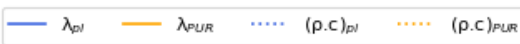

Figure 11: Sensitivity analysis of the 18R15C model for the lightweight wall during the sunny day.

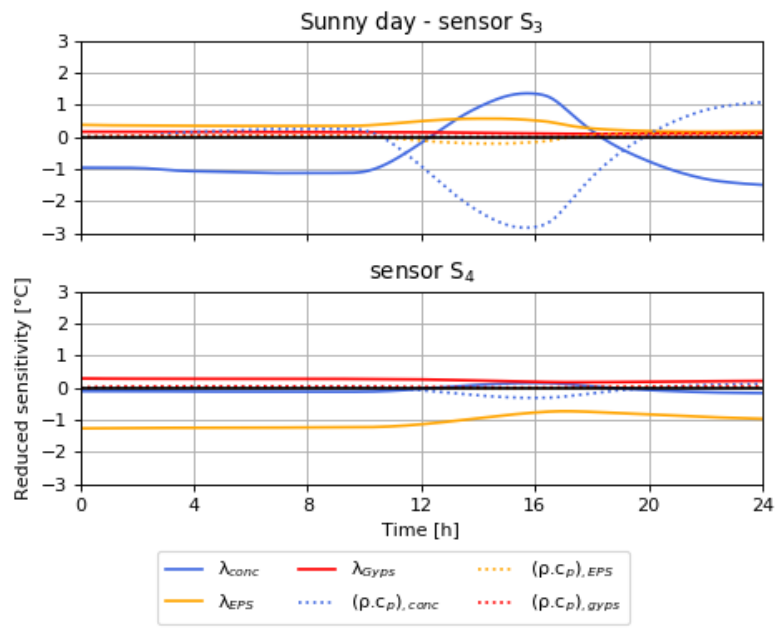

Figure 12: Sensitivity analysis of the 18R15C model for the heavyweight wall during the sunny day.

Regarding the sensitivity analysis for the heavyweight weight wall in Figure $12, \lambda_{\text {conc }}$ and $(\rho . c)_{\text {conc }}$ show high sensitivities but they seem correlated. The sensitivity of $\lambda_{\text {conc }}$ is not negligible.

\section{Correlation matrices}

The analysis of correlation matrices gives useful information for identification: if the parameters are strongly correlated, it will be impossible to identify all of them at the same time.

Table 3 shows the correlation matrices of the $18 R 15 C$ model for the lightweight wall during the sunny day. The results for the $Q R$ model and the $R F$ model are close to those shown in Table 3. The results of correlation matrices show that parameters are strongly correlated since values are close to \pm 1 , mainly for sensor $S_{4}$ (cells are coloured in light grey when absolute value is above $0.8)$. Since correlation was strong between parameters, it was impossible to estimate all the parameters at the same time. That is the reason why at least two sensors need to be used to possibly decorrelate the parameters. Moreover, it was intended to carry out a partial identification of the $R$ - and $C$-values.

Table 3: Correlation matrix for the sunny day (18R15C model) - Lightweight wall.

\begin{tabular}{c|cccc}
\hline \multicolumn{5}{|c}{ Sensor S3 } \\
\hline & $\lambda_{P U R}$ & $\lambda_{p l}$ & $(\rho . c)_{P U R}$ & $(\rho . c)_{p l}$ \\
\hline$\lambda_{P U R}$ & 1 & 0.15 & 0.02 & 0.47 \\
$\lambda_{p l}$ & 0.15 & 1 & 0.96 & 0.87 \\
$(\rho . c)_{P U R}$ & 0.02 & 0.96 & 1 & 0.73 \\
$(\rho . c)_{p l}$ & 0.47 & 0.87 & 0.73 & 1 \\
\hline & \multicolumn{5}{|c}{ Sensor $\mathbf{S}_{\mathbf{4}}$} \\
\hline$\lambda_{P U R}$ & 1 & 1 & 1 & 1 \\
$\lambda_{p l}$ & 1 & 1 & 1 & 1 \\
$(\rho . c)_{P U R}$ & 1 & 1 & 1 & 1 \\
$(\rho . c)_{p l}$ & 1 & 1 & 1 & 1
\end{tabular}

In the case of the lightweight wall, we assumed that $R$ value is mostly impacted by $\lambda_{P U R}$ (about $94 \%$ of total) and $C$-value is largely impacted by ( $\rho . c)_{p l}$ (about $91 \%$ of total). In the case of the heavyweight wall, we assumed that $R$ value is mostly impacted by $\lambda_{E P S}$ (about $93 \%$ of total) and $C$-value is largely impacted by ( $\rho . c)_{\text {conc }}$ (about $93 \%$ of total).

\section{Estimation of uncertainties}

Different sources of uncertainties were analysed:

- the uncertainty due to the inverse technique,

- the uncertainty due to the measurement noise of temperature sensors and the measurement of boundary conditions.

In order to evaluate these uncertainties, a white noise and an offset were applied to all boundary conditions and temperature measurements. The standard deviation of the white noise for temperature measurements is $\pm 0.15^{\circ} \mathrm{C}$ (two times the standard deviation evaluated during calibration). The standard deviation of the white noise for the solar radiation measurement is $\pm 5 \mathrm{~W} \cdot \mathrm{m}^{-2}$. As an initial approach, the offset of both types of measurements are included in a range smaller than the one of standard deviations, i.e., $\pm 0.1^{\circ} \mathrm{C}$ for temperature measurements and $\pm 1 \mathrm{~W} \cdot \mathrm{m}^{-2}$ for the solar radiation measurement. More than 100 tests were performed for which offsets were randomly selected.

The difference between the theoretical $R$ - and $C$-values and the estimated $R$ - and $C$-values were considered as the uncertainty due to the inverse technique, the model and the measurement noise.

\section{Application to simulated data of a lightweight wall}

In this part, values of $T^{\text {sensor }}$ in equation 7 were simulated experimental data with or without noise. Boundary conditions during this test are shown in Figure 6. Identification was applied on sensors $\mathrm{S}_{3}$ and $\mathrm{S}_{4}$. The $R$ and $C$-values were estimated at the same time by identifying $\lambda_{P U R}$ and $(\rho . c)_{p l}$.

The accuracy of the identification technique using a given model was assessed by comparing differences between the identified values and $R_{\text {Light }}$ and $C_{\text {Light }}$. Results are plotted in Figure 13. 
Regarding identification applied to simulated experimental data without noise, the $Q R$ model is the most accurate in estimating both $R$ - and $C$-values. When applying the measurement noise, the difference between the identified values and the theoretical values is in \pm 0.4 $\mathrm{m}^{2} . \mathrm{K} . \mathrm{W}^{-1}$ for the $R$-value and in $\pm 5 \mathrm{~kJ} \cdot \mathrm{m}^{-2} \cdot \mathrm{K}^{-1}$ for the $C$ value for all the models. In this case, the standard deviation of the difference is lower the $Q R$ model.

To conclude on the uncertainty due to the inverse technique, the noise related to model choices and measurement is defined as the mean of the difference between the theoretical values and the identified values in the case of noised measurements plus two times its standard deviation so that $95 \%$ of future estimations are included. This uncertainty equals $0.29 \mathrm{~m}^{2} . \mathrm{K}^{-\mathrm{W}^{-1}}$ for the $R$-value and $8 \mathrm{~kJ} . \mathrm{m}^{-2} \cdot \mathrm{K}^{-1}$ for the $C$-value for the $18 R 15 C$ model, $0.30 \mathrm{~m}^{2} . \mathrm{K} \cdot \mathrm{W}^{-1}$ for the $R$-value and $6 \mathrm{~kJ} \cdot \mathrm{m}^{-2} \cdot \mathrm{K}^{-1}$ for the $C$-value for the $R F$ model and $0.33 \mathrm{~m}^{2} . \mathrm{K}^{-\mathrm{W}^{-1}}$ for the $R$-value and $3 \mathrm{~kJ} \cdot \mathrm{m}^{-2} \cdot \mathrm{K}^{-1}$ for the $C$-value for the $Q R$ model.
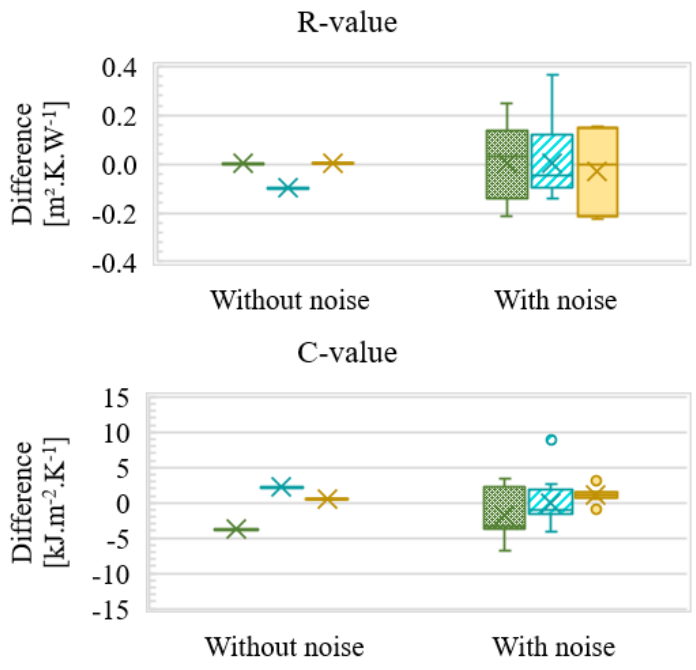

18R15C model $\square \mathrm{RF}$ model $\square \mathrm{QR}$ model

Figure 13: Estimation of $R$ - and $C$-values for the lightweight wall - data with and without noise.

\section{Application to simulated data of a heavyweight wall}

In this part, values of $T^{\text {sensor }}$ in equation 7 were simulated experimental data with or without noise. Boundary conditions during this test are shown in Figure 6 . Identification was applied on sensors $\mathrm{S}_{3}$ and $\mathrm{S}_{4}$. The $R$ and $C$-values were estimated at the same time by identifying $\lambda_{E P S}$ and ( $\left.\rho . c\right)_{\text {conc }}$.

The accuracy of the identification technique using a given model was assessed by comparing differences between the identified values and $R_{\text {Heavy }}$ and $C_{\text {Heavy }}$ Results are plotted in Figure 14. Accuracy of the $R$ - and $C$-values assessment is high for the $Q R$ model regarding simulated experimental data without noise. As in the lightweight wall case, the 18R15C model is better in identifying the $R$-value than the $C$-value.

In the case of the heavyweight wall, the uncertainty due to the inverse technique, the model and the measurement noise equals $\pm 0.19 \mathrm{~m}^{2} . \mathrm{K} . \mathrm{W}^{-1}$ for the $R$-value and \pm 15
$\mathrm{kJ} . \mathrm{m}^{-2} \cdot \mathrm{K}^{-1}$ for the C-value for the 18R15C model, \pm 0.21 $\mathrm{m}^{2} . \mathrm{K} . \mathrm{W}^{-1}$ for the $R$-value and $\pm 10 \mathrm{~kJ} \cdot \mathrm{m}^{-2} \cdot \mathrm{K}^{-1}$ for the $C$ value for the $R F$ model and $\pm 0.30 \mathrm{~m}^{2} . \mathrm{K}^{-W^{-1}}$ for the $R$ value and $\pm 10 \mathrm{~kJ} \cdot \mathrm{m}^{-2} \cdot \mathrm{K}^{-1}$ for the $C$-value for the $Q R$ model.
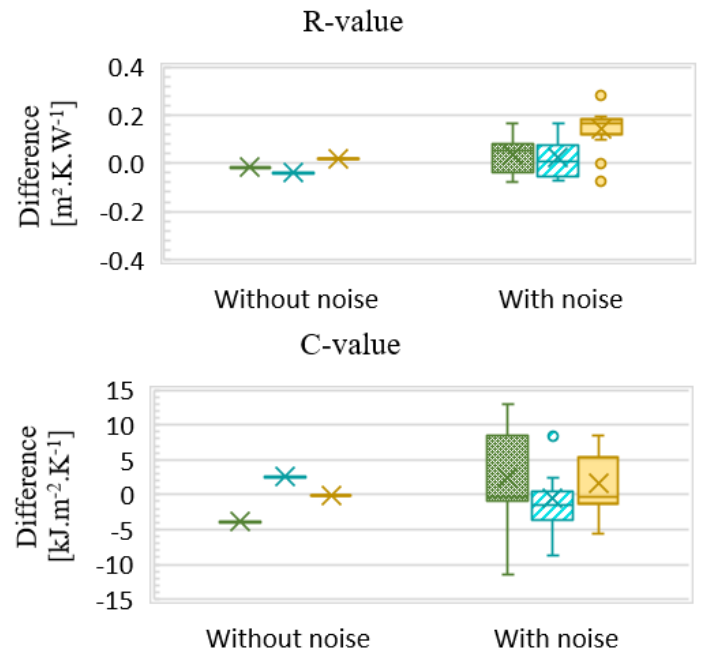

18R15C model $\square \mathrm{RF}$ model $\square$ QR model

Figure 14: Estimation of $R$ and $C$-values for the heavyweight wall - data with and without noise.

Comparing the lightweight wall and the heavyweight wall studies, the estimation of the $R$-value is better in the heavyweight wall case whereas the estimation the $C$-value is better in the lightweight wall case.

\section{Application to experimental data from the first component}
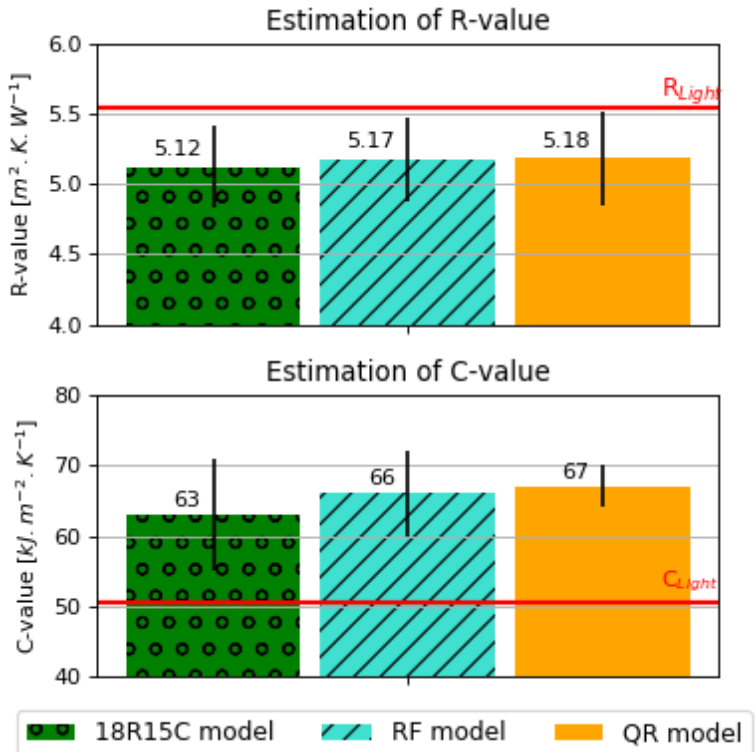

Figure 15: Estimation of $R$ and $C$-values of the lightweight component from experimental data.

In this part, values of $T$ sensor in equation 7 and boundary conditions were experimental data measured during the ROLBS case by temperature sensors and the weather station located in La Rochelle, France. Boundary conditions during this test are shown in Figure 6. The first 
38 hours were used as initialization period. Identification techniques were applied on data of the next 184 hours.

Figure 15 shows the estimation of $R$ - and $C$-values of the lightweight wall. The $R$-value is estimated at around 5.2 $\mathrm{m}^{2} . \mathrm{K} . \mathrm{W}^{-1}$ by all models and the $C$-value is estimated between 63 and $67 \mathrm{~kJ} \cdot \mathrm{m}^{-2} \cdot \mathrm{K}^{-1}$. If the uncertainty defined above is taken into consideration, the $R$-value is estimated between 4.9 and $5.5 \mathrm{~m}^{2} \cdot \mathrm{K}^{-1} \mathrm{~W}^{-1}$ and the $\mathrm{C}$-value is estimated between 55 and $72 \mathrm{~kJ} \cdot \mathrm{m}^{-2} \cdot \mathrm{K}^{-1}$.

\section{Conclusion}

In this article, the one-dimensional heat transfer in a multilayer wall instrumented with temperature sensors under real weather conditions was studied. A comparison of the performance in identifying thermal characteristics of walls of different models were proposed.

First, the 6R3C model was rejected since its accuracy was not satisfying. Concerning the temperature sensors, a sensitivity analysis was completed with the aim of rejecting the sensors that were too sensitive to parameters defined with a low accuracy, such as the internal and external convective and radiative heat transfer coefficients and the exterior solar surface absorptivity. Only 2 sensors out of 5 were finally useful. Then, inverse techniques were applied on two walls. Their objective was to precisely assess the thermal characteristics of walls on site and on experimental data. A first approach was presented to take into consideration the noise of measurements. This point is fundamental to determine the quality of the estimation. Regarding results on the lightweight wall, all the models could estimate the $R$ value with an uncertainty lower than $\pm 0.33 \mathrm{~m}^{2} . \mathrm{K}^{\mathrm{W}} \mathrm{W}^{-1}$ and the $C$-value with an accuracy lower than $\pm 8 \mathrm{~kJ} \cdot \mathrm{m}^{-2} \cdot \mathrm{K}^{-1}$. For the heavyweight wall, all the models could estimate the $R$-value with an uncertainty lower than \pm 0.3 $\mathrm{m}^{2} . \mathrm{K} \cdot \mathrm{W}^{-1}$ and the $C$-value with an accuracy lower than $\pm 15 \mathrm{~kJ} \cdot \mathrm{m}^{-2} \cdot \mathrm{K}^{-1}$.

This method was applied on experimental data. The estimated $R$-value is $20 \%$ lower than the theoretical value. The estimated $C$-value is $30 \%$ higher than the theoretical value. This can be explained by the risk that the wall built does not fully correspond to the design choices. This method could also be applied on heavyweight walls since a satisfying accuracy was obtained on the estimation of the $R$ - and $C$-values.

\section{Acknowledgement}

The activities have been carried out in the framework of the Research Project "CITEE - Innovative components for building envelopes", financed by the European Union and the French region Nouvelle-Aquitaine with the industrial partners TIPEE Platform and EDF.

\section{References}

Beck, J.V., and Arnold, K.J. (1977). Parameter Estimation in Engineering and Science. Wiley (USA).
Beck, J.V., Blackwell, B., and St. Clair, C.R. (1985). Inverse Heat Conduction: Ill-Posed Problems. Wiley (USA).

COMSOL (2019). Introduction to COMSOL Multiphysics.

Deconinck, A.-H., and Roels, S. (2016). Comparison of characterisation methods determining the thermal resistance of building components from onsite measurements. Energy and Buildings 130, 309-320.

Eleftheriadis, G., and Hamdy, M. (2017). Impact of building envelope and mechanical component degradation on the whole building performance: a review paper. Energy Procedia 132, 321-326.

Gaspar, K., Casals, M., and Gangolells, M. (2016). A comparison of standardized calculation methods for in situ measurements of façades U-value. Energy and Buildings 130, 592-599.

ISO 9869-1 (2014). Thermal insulation - Building elements - In-situ measurement of thermal resistance and thermal transmittance - Part 1: Heat flow meter method.

Maillet, D., André, S., Batsale, J.-C., Degiovanni, A., and Moyne, C. (2000). Thermal quadrupoles: solving the heat equation through integral transforms. Wiley (Great Britain).

Meiss, A., and Feijo-Munoz, J. (2015). The energy impact of infiltration: a study on buildings located in north central Spain. Energy Efficiency 8, 51-64.

Mitalas, G.P., and Stephenson, D.G. (1967). Room thermal response factors. National Research Council of Canada 342.

Nelder, J.A., and Mead, R. (1965). A Simplex Method for Function Minimization. Computer Journal 7, 308313.

NF EN 12667 (2001). Thermal performance of building materials and products - Determination of thermal resistance by means of guarded hot plate and heat flow meter methods - Products of high and medium thermal resistance.

NF EN 12939 (2000). Thermal performance of building materials and products - Determination of thermal resistance by means of guarded hot plate and heat flow meter methods - Thick products of high and medium thermal resistance.

NF EN ISO 6946 (2017). Building components and building elements - Thermal resistance and thermal transmittance - Calculation method.

Remy, B., and Degiovanni, A. (2005). Parameters estimation and measurement of thermophysical properties of liquids. International Journal of Heat and Mass Transfer 48,4103-4120. 